\title{
Implicación de los alumnos del Grado de Podología en la innovación docente de la Microbiología
}

\author{
María Luisa Gómez-Lus ${ }^{1}$; David Sevillano²; Natalia González³; Luis Alou ${ }^{4}$
}

Fecha de recepción: 11 de diciembre de 2018 / Fecha de aceptación: 2 de abril 2019.

Resumen. Se ha planteado como objetivo la mejora de la calidad de la docencia de la Microbiología mediante la actualización de la metodología docente, introduciendo como actividad docente el aprendizaje activo basado en preguntas (inquiry-based learning:IBL) para conseguir mejorar las competencias que deberán adquirir los estudiantes como parte de su formación integral. En este estudio han participado 63 alumnos de Segundo Curso del Grado de Podología de la Universidad Complutense de Madrid (Curso 2017-2018), y se ha calculado el porcentaje de alumnos que participaron en las 4 pruebas (4IBL), 3 pruebas (3 IBL), en 2 (2 IBL), en 1 (1 IBL), y los que no participaron en ninguna y posteriormente se relacionó con las calificaciones obtenidas en la asignatura de Microbiología. Se incluyeron las preguntas IBL que se realizaron en clase en el campus virtual de la asignatura, pero incluyendo la corrección de las mismas.

En los alumnos que realizaron alguna prueba IBL se obtuvieron calificaciones mejores que en aquellos que no habían participado en ninguna. Consideramos que el uso del campus virtual unido a la actualización en la metodología docente puede mejorar el rendimiento académico de los estudiantes de Microbiología.

Palabras clave: Microbiología; Podología; Aprendizaje activo; Campus Virtual; Rendimiento;

\section{[en] Implication of the students of the Degree of Podiatry in the teaching innovation of Microbiology}

Abstract. Our focus here is to improve teaching methods in University microbiology classes and the development of educational practices that explicit engage students while enhacing how they learn Microbiology. These practices including inquiry-based learning (IBL) in which students are actively engaged in the learning process for improving the skills to be acquired as part of their effective learning. This study included 63 Microbiology students of Second Year Degree in Podiatry at the Complutense University (Academic Year 2017-2018), and calculated the percentage of students who used these resources 4 IBL, 3 IBL , 2IBL, 1IBL, and that did not participate in any and subsequently related to the student performance in the Microbiology exam. The IBL questions that were done in class, were included in the virtual campus, including the correction .

Students who prepared IBL scored higher en exam questions, findings also indicate that this activity can measure learning and improve exam preparation. We believe that the benefits of active learning methods with virtual campus over lecture instruction are consistent and can increases student performance in Microbiology.

Keywords: Microbiology; Podiatry; Active Learning; Virtual Campus; Performance;

Sumario: 1. Introducción. 2. Material y métodos. 3. Resultados y discusión. 4. Bibliografía.

Área de Microbiología, Departamento de Medicina, Facultad de Medicina. Universidad Complutense de Madrid. Avenida Complutense s/n. 28040 Madrid. España

mlgomezl@ucm.es

2 Área de Microbiología, Departamento de Medicina, Facultad de Medicina. Universidad Complutense de Madrid. dsevill@med.ucm.es

3 Área de Microbiología, Departamento de Medicina, Facultad de Medicina. Universidad Complutense de Madrid. natgonzalez@med.ucm.es

4 Área de Microbiología, Departamento de Medicina, Facultad de Medicina. Universidad Complutense de Madrid. luisalou@med.ucm.es 
Cómo citar: Gómez-Lus, M. L. et ali (2019) Implicación de los alumnos del Grado de Podología en la innovación docente de la Microbiología, en Revista internacional de ciencias podológicas 13(1), 55-60.

Los autores declaran no tener ningún tipo de interés económico o comercial.

\section{Introducción}

El conocimiento de la Microbiología es básico para establecer una bases que permitan el diagnóstico y el tratamiento de las infecciones desde una perspectiva teórica y práctica, así cómo el seguimiento del aprendizaje de los alumnos (1). El objetivo de este trabajo es comparar los resultados obtenidos en el curso 2017-2018 con el aprendizaje reflexivo mediante preguntas IBL (Inquired Based Learning) de manera presencial y en relación al rendimiento académico de los alumnos en la asignatura de Microbiología del Grado de Podología de la Universidad Complutense de Madrid (UCM) y también se ha valorado la progresión de la participación de los alumnos, presencial y no presencial, desde que se instauraron en el curso 2011-2012, las nuevas líneas de trabajo de innovación educativa en la docencia de la Microbiología en el Grado de Podología y en el Grado de Medicina $(2,3)$. Por otra parte se ha comprobado cómo este tipo de aprendizaje incrementa el rendimiento académico mejorando las calificaciones de diferentes asignaturas (4 ) y es necesario demostrar su eficacia para producir un cambio en la metodología docente (5).

\section{Material y métodos}

En el estudio participaron 63 alumnos, que cursaban Microbiología durante el primer cuatrimestre del Curso 2017-2018, en segundo curso del Grado de Podología de la Universidad Complutense de Madrid.

Al inicio de la exposición de un grupo de 3 temas se entregaba una hoja de trabajo en el aula en la que se realizaba una prueba de selección compuesta por 15 preguntas a las que el alumno podía contestar si lo que se afirmaba era verdadero o falso y aportaba información sobre el conocimiento previo sobre el tema; esa hoja de trabajo permitía a los alumnos trabajar para autoevaluar su propio aprendizaje, cómo autoevaluación diagnóstica.

Al finalizar esa unidad temática, contestaban de nuevo esas preguntas en clase y valoraban los aciertos conseguido antes de la primera clase y después de última clase, cómo autoevaluación formativa, de cada grupo de temas. Se realizaron 4 pruebas de 15 preguntas IBL, correspondientes a preguntas de Estructura y Genética (IBL-A), Bacteriología I (Cocos Gram positivos) (IBL-B), Bacteriología II (Bacilos Gram negativos) (IBL-C) y Micología y Parasitología (IBL-D).

Posteriormente las hojas de trabajo se colgaban en el campus virtual, después de responderlas y discutirlas en clase pero incluyendo la corrección de las mismas, junto con los resúmenes de cada uno de los temas que componían la unidad temática.

Se ha calculado el porcentaje de alumnos que participaron en las 4 pruebas (4 IBL), en 3 (3 IBL) en 2 ( 2 IBL), en 1 ( 1 IBL) $\mathrm{y}$ los que no participaron en ninguna (no IBL). Posteriormente se relacionó con las calificaciones obtenidas en la asignatura de Microbiología, se empleó el test de chicuadrado. La participación en todas las pruebas era voluntaria y no se tenía en cuenta para la calificación final. Se empleó el test de chi-cuadrado para comporar la evolución de la participación presencial de la realización de las 4 pruebas IBL.

\section{Resultados y discusión}

Del total de 63 alumnos, 14 alumnos $(22,2$ $\%)$ participaron y entregaron corregidos las cuatro tandas de (4 IBL), 23 alumnos (36,5 $\%)$ las tres tandas de IBL (3 IBL), 10 (15,9 $\%$ ) alumnos lo hicieron con dos tandas de IBL (2 IBL) y $5(7,9 \%)$ alumnos entregaron solamente una (1 IBL), mientras que 11 alumnos $(17,5 \%)$ no entregaron ningún IBL (Tabla I y Figura 1). 


\begin{tabular}{|c|c|c|c|c|c|c|c|}
\hline & TOTAL & $\begin{array}{c}\text { ALGUN } \\
\text { IBL }\end{array}$ & 4 IBL & 3 IBL & 2 IBL & 1 IBL & NO IBL \\
\hline Sobresaliente & $4(6,3)$ & $3(4,8)$ & $0(0,0)$ & $2(3,2)$ & $1(1,6)$ & $0(0,0)$ & $1(1,6)$ \\
\hline Notable & $10(15,9)$ & $10(15,9)$ & $2(3,2)$ & $6(9,5)$ & $0(0,0)$ & $2(3,2)$ & $0(0,0)$ \\
\hline Aprobado & $24(38,1)$ & $21(33,3)$ & $7(11,1)$ & $10(15,9)$ & $3(4,8)$ & $1(1,6)$ & $3(4,8)$ \\
\hline Suspenso & $18(28,6)$ & $16(25,4)$ & $3(4,8)$ & $5(7,9)$ & $6(9,5)$ & $2(3,2)$ & $2(3,2)$ \\
\hline $\begin{array}{c}\text { No } \\
\text { presentados }\end{array}$ & $7(11,1)$ & $2(3,2)$ & $2(3,2)$ & $0(0,0)$ & $0(0,0)$ & $0(0,0)$ & $5(7,9$ \\
\hline \begin{tabular}{c} 
Total \% \\
\hline
\end{tabular} & $63(100,0)$ & $52(82,5)$ & $14(22,2)$ & $23(36,5)$ & $10(15,9)$ & $5(7,9)$ & $11(17,5)$ \\
\hline
\end{tabular}

Tabla I: Calificaciones obtenidas en relación con la participación en Inquired Based Learning (IBL)

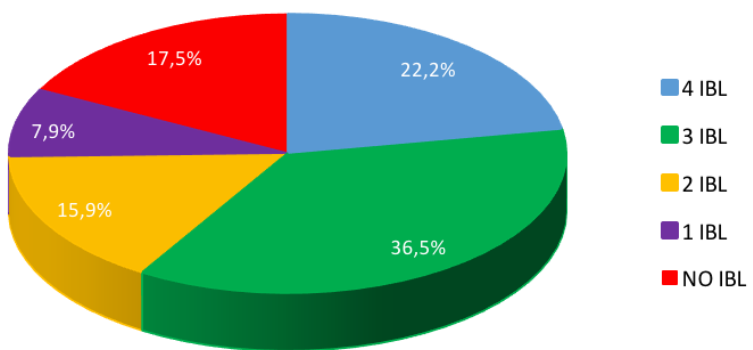

Figura 1 : Participación en realización presencial de IBL

Los alumnos que realizaron 4 IBL, $2(3,2$ $\%)$ notable, y $7(11,1 \%)$ aprobado, $3(4,8 \%)$ suspenso y 2 no presentado (3,2\%).

Los alumnos que realizaron 3 IBL obtuvieron $2(3,2 \%)$ sobresaliente, y $6(9,5$ $\%)$ notable, 10 aprobado $(15,9 \%), 5$ suspenso $(7,9 \%)$ y del grupo que realizaron 2 IBL, obtuvieron sobresaliente 1 alumno $(1,6 \%)$, notable $3(4,8 \%)$, suspenso $6(9,5 \%)$ del grupo que realizó 1 IBL las calificaciones fueron 2
$(3,2 \%)$ notable, $1(1,6 \%)$ aprobado y $2(3,2$ $\%)$ suspenso.

Del grupo de alumnos que no presentaron ningún IBL, las calificaciones fueron: 1 (1,6 $\%)$ sobresaliente, $3(4,8 \%)$ aprobado y $2(3,2$ $\%)$ suspenso. En cuanto a los no presentados, destaca que 2 alumnos realizaron 4 IBL, pero la mayoría, 5 alumnos no realizaron ninguno (Figura 2).

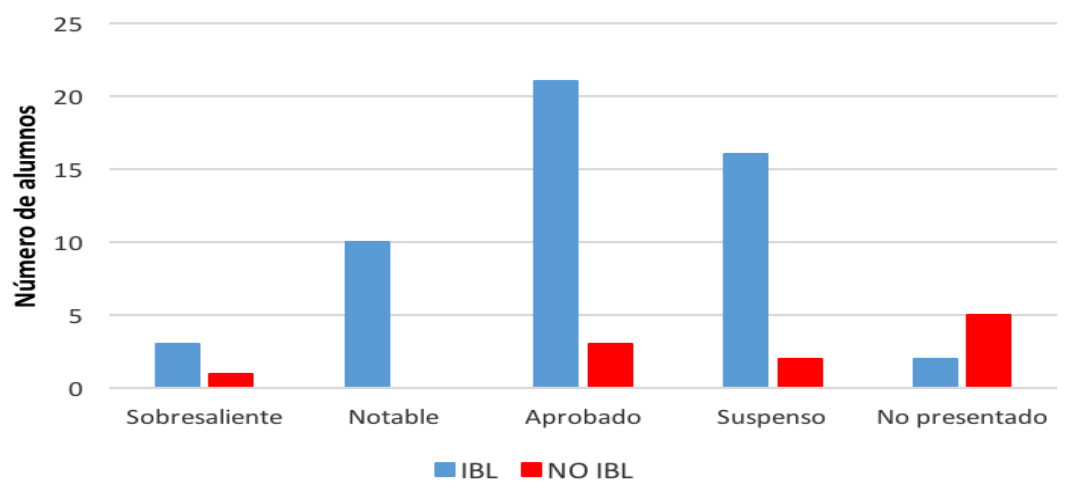

Figura 2: Relación entre calificaciones y realización de IBL. 
Posteriormente se relacionó con las calificaciones obtenidas en la asignatura de Microbiología, se empleó el test de chicuadrado, demostrando que los alumnos que realizaron 3 y 4 IBL obtuvieron un mayor rendimiento académico $(\mathrm{p}=0,0143)$ que los que realizaron menos o ninguno (Figura 3 ).

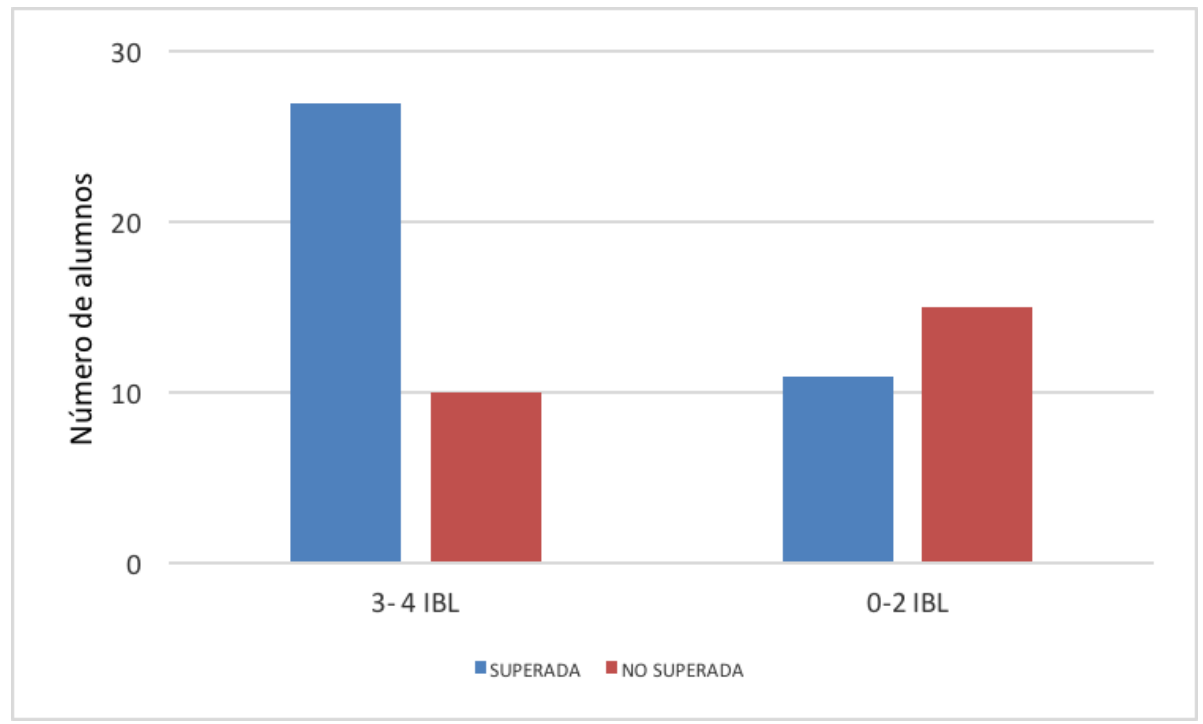

Figura 3: Relación entre calificaciones y realización de IBL.

En relación a la participación en los diferentes IBL, comenzó con IBL-A con un $74,6 \%$ (47 alumnos), seguido de IBL-B un $68,3 \%$ (43 alumnos), IBL-C un 58,7 \% (37 alumnos), y en la última IBL-D se redujo hasta un 38,1 \% (24 alumnos), (Figura 4) siendo estas diferencias estadísticamente significativas $(\mathrm{p}=0,0002)$. Pese a que la participación media de un $59,9 \%$ el interés por realizar la actividad de modo presencial decayó progresivamente.

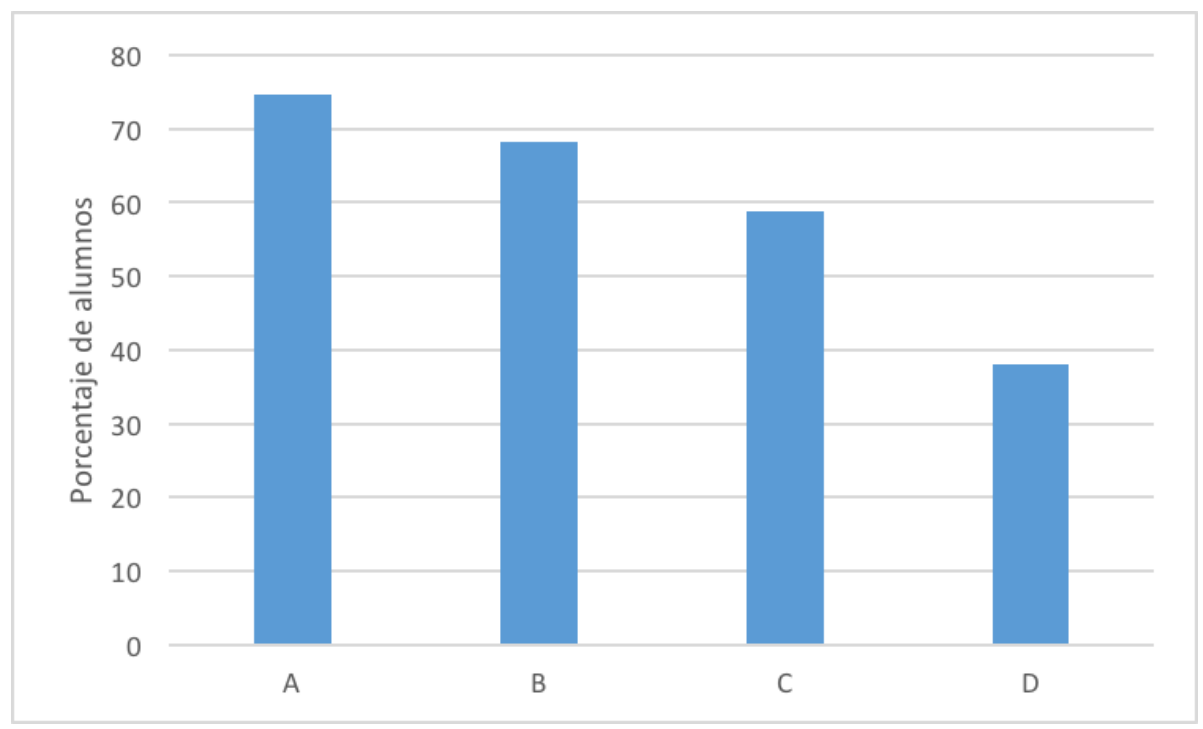

Figura 4: Evolución de la participación presencial de realización de las 4 pruebas IBL. 


\begin{tabular}{|c|c|c|c|}
\hline $\begin{array}{c}\text { Número de visitas a los } \\
\text { temas } \\
(\text { media } \pm \text { DS })\end{array}$ & IBL & $\begin{array}{c}\text { Número de visitas a los } \\
\text { IBL sin corregir }\end{array}$ & $\begin{array}{c}\text { Número de visitas a los } \\
\text { IBL corregidos }\end{array}$ \\
\hline \multirow{5}{*}{$162 \pm 30$} & A & 153 & 113 \\
\cline { 2 - 4 } & B & 98 & 89 \\
\cline { 2 - 4 } & C & 73 & 92 \\
\cline { 2 - 4 } & D & 51 & 79 \\
\hline
\end{tabular}

Tabla II: Participación no presencial en el campus virtual

Las tecnologías informáticas están destinadas a seguir desempeñando un papel fundamental en la renovación metodológica ya que el desarrollo informático permite a profesores y estudiantes un acceso rápido a la información a través de las tecnologías de la información y la comunicación (TIC), las plataformas educativas y otros recursos electrónicos por lo que complementamos el trabajo IBL en clase con la inclusión en el campus virtual de la UCM de las hojas de trabajo antes de la clase y después de la clase corregidas así cómo los temas impartidos (Tabla II), en los que se observa una mayor número de vistas a las clases impartidas (162 $\pm 30)$ que las hojas de trabajo IBL sin corregir $(93,7 \pm 40,9)$ y corregidas $(93,2 \pm 14,3)$.

La evaluación realizada con la metodología docente mediante las hojas de trabajo IBL, de tipo diagnóstica $\mathrm{y}$ formativa, supone un feedback dinámico entre el profesor $\mathrm{y}$ los alumnos, permitiendo la reflexión, y teniendo cómo propósito alcanzar un nivel de comprensión sobre el programa educativo propuesto en Microbiología y sobre los efectos que está generando en el conocimiento de la asignatura por parte de los alumnos, identificando su propio aprendizaje de manera continua y dinámica, apreciando sus logros, y también la posibilidad de realizar un inventario de conceptos sobre todo cuando se realiza de modo presencial ya que favorece las destrezas colaborativas en los grupos y las actitudes y aptitudes para la comunicación. Pero hay muchos alumnos que por diversos motivos no pueden asistir a las clases, por lo que las hojas de trabajo colgadas en el campus virtual, así cómo los temas impartidos han permitido el aprendizaje autónomo, de modo no presencial, respetando la pluralidad del alumnado.
En el curso 2017-2018, al comparar los resultados académicos de los alumnos de Microbiología de segundo curso de Podología, se observa cómo los estudiantes que realizaron voluntariamente las cuatro pruebas IBL obtuvieron las calificaciones más altas, sobresaliente y notable, y el mayor número de suspensos y no presentados se encontraba en el grupo de los alumnos que habían participado menos coincidiendo con otros autores que obtenían resultados similares(6). Por otra parte únicamente el 17,5\% de los alumnos no realizó ningún IBL, por lo que se está incrementando cada curso el grado de participación, ya que en el primer curso en el que se implementó, 2010-2011, solamente rellenaron las hojas de trabajo IBL un $19,60 \%$, ya que la mayoría de las clases se impartían mediante lección magistral y era una minoría de alumnos a la que conseguíamos motivar con los métodos de aprendizaje centrados en el alumno, por lo que estamos integrando con éxito este tipo de docencia que mejora el aprendizaje presencial y no presencial (7).

La inclusión de resúmenes de las clase y las hojas IBL corregidas en el campus cómo sistema virtual ha permitido consultar el material didáctico de Microbiología ,que ha compensado la falta de asistencia a clase, superando la asignatura mediante la flexibilización de las trayectorias de aprendizaje. Así nos planteamos como reto motivar a los alumnos en la metodología de la participación tanto presencial cómo no presencial, ya que el interés por las actividades presenciales va disminuyendo durante el transcurso del curso,pero pueden seguir trabajando a través del campus virtual con la realización de tutorías presenciales o no presenciales que flexibilicen el aprendizaje. 


\section{Bibliografía}

1. Chaudhry MA. 2011. Assessment of Microbiology students' progress with an audience response system, Journal of Microbiology Biology Education; 12.2: (200-201).

2. Gómez-Lus ML, Ramos C, Alou L, Prieto J. 2016.El aprendizaje activo de la Microbiología en el Grado de Podología incrementa el rendimiento académico. Revista Internacional de Ciencias Podológicas; 10, (85-94)

3. Gómez-Lus Centelles ML, Alou L, Prieto J. 2018. Integrando el aprendizaje activo de la Microbiología en el Grado de Medicina: evolución de la implicación de los estudiantes. Educación Médica; 19 (2): (77-81).

4. Freeman $\mathrm{S}$ et al. 2014. Active learning increases student performance in science, engineering, and mathematics. Proceedings of the National Academy of Sciences of the United States of America; 111: 8410-8415.

5. Cooper K, Ashley M, Brownell S. 2017.Using expectancy value theory as a framework to reduce student resistance to active learning: a proof of concept. Journal of Microbiology Biology Education; 18, 2: (1-7).

6. Marbach-Ad G, Rietschel CH, Saluja N, Carleton KL, Haag ES. 2016. The Use of Group Activities in Introductory Biology Supports Learning Gains and Uniquely Benefits High-Achieving Students. Journal of Microbiology Biology Education;17(3): (360-369).

7. Wieman C E. 2014.Large-scale comparison of science teaching methods sends clear message. Proceedings of the National Academy of Sciences of the United States of America; 111: (8319-8320). 